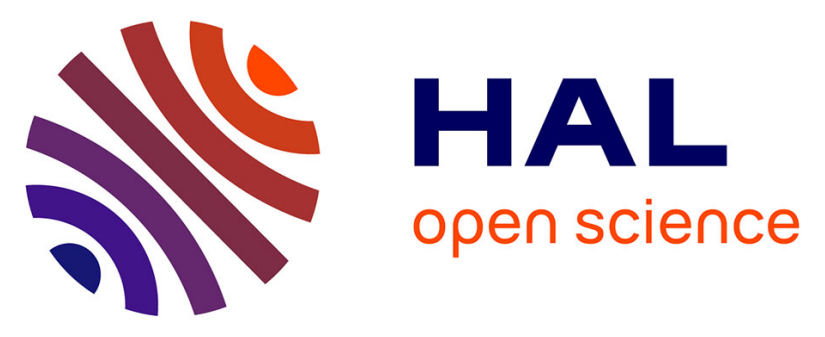

\title{
Circular Dielectric Rod with Conformal Strip of Graphene as Tunable Terahertz Antenna: Interplay of Inverse Electromagnetic Jet, Whispering Gallery and Plasmon Effects
}

Sergii V. Dukhopelnykov, Mario Lucido, Ronan Sauleau, Alexander I. Nosich

\section{To cite this version:}

Sergii V. Dukhopelnykov, Mario Lucido, Ronan Sauleau, Alexander I. Nosich. Circular Dielectric Rod with Conformal Strip of Graphene as Tunable Terahertz Antenna: Interplay of Inverse Electromagnetic Jet, Whispering Gallery and Plasmon Effects. IEEE Journal of Selected Topics in Quantum Electronics, 2021, 27 (1), pp.4600908. 10.1109/JSTQE.2020.3022420 . hal-03003558

\section{HAL Id: hal-03003558 https://hal.science/hal-03003558}

Submitted on 1 Dec 2020

HAL is a multi-disciplinary open access archive for the deposit and dissemination of scientific research documents, whether they are published or not. The documents may come from teaching and research institutions in France or abroad, or from public or private research centers.
L'archive ouverte pluridisciplinaire HAL, est destinée au dépôt et à la diffusion de documents scientifiques de niveau recherche, publiés ou non, émanant des établissements d'enseignement et de recherche français ou étrangers, des laboratoires publics ou privés. 


\title{
Circular Dielectric Rod with Conformal Strip of Graphene as Tunable Terahertz Antenna: Interplay of Inverse Electromagnetic Jet, Whispering Gallery and Plasmon Effects
}

\author{
Sergii V. Dukhopelnykov, Member, IEEE, Mario Lucido, Senior Member, IEEE, Ronan Sauleau, \\ Fellow, IEEE, and Alexander I. Nosich, Fellow, IEEE
}

\begin{abstract}
We consider the radiation characteristics of a $\mathrm{THz}$ antenna made of a circular dielectric rod decorated with conformal graphene strip and illuminated by the field of a line magnetic current. The strip has arbitrary angular size and location and its surface impedance is characterized with Kubo theory. Our mathematically accurate analysis uses a dedicated hypersingular integral equation for the current induced on the strip. Discretization of this equation is carried out by the Nystrom-type method, which has a guaranteed convergence. We study the dependences of the powers radiated and absorbed in this configuration and also the directivity of antenna emission, in wide frequency range from 0 to $10 \mathrm{THz}$. They show very interesting interplay between the broadband inverse photonic-jet effect of lens-like dielectric rod and two types of resonances: on the moderate-Q plasmon modes of graphene strip and on the extremely high-Q whispering-gallery modes of the circular rod.
\end{abstract}

Index Terms - circular dielectric rod, graphene strip, line current, integral equation, plasmon, inverse photonic jet

\section{INTRODUCTION}

$\mathrm{B}$ $\mathrm{OTH}$ in $\mathrm{THz}$ and shorter frequency ranges, a ubiquitous element of almost every sensor is an integrated lens antenna, which concentrates the incoming electromagnetic waves on a miniature receiving circuit. Perhaps, the simplest design of a lens is a uniform optically transparent sphere or, in two-dimensional (2-D) case, circular cylinder, or rod. This is because such a sphere or a rod produces a remarkable near-field phenomenon, which at first obtained the name "nanojet" and later became known as "photonic jet" [12]. In fact, it should be, probably, called "electromagnetic jet" (EMJ), because this phenomenon can be found in all frequency ranges, if only the relative dielectric permittivity of sphere or rod is $\varepsilon<4$ and its radius is $R \geq 2 \lambda$, where $\lambda$ is the freespace wavelength [1,2]. If the radius gets larger, EMJ

Manuscript received March 31, 2020. This work was supported, in part, by the National Research Foundation of Ukraine, project no 2020.02.0150.

S. V. Dukhopelnykov and A. I. Nosich are is with the Laboratory of Micro and Nano Optics, Institute of Radio-Physics and Electronics NASU, Kharkiv, 61085, Ukraine (e-mail: dukh.sergey@gmail.com and anosich@yahoo.com).

M. Lucido is with the DIEI, University of Cassino and Southern Lazio, Cassino, Italy (email: lucido@unicas.it)

R. Sauleau is with IETR, Universite de Rennes 1, Rennes, 35042, France (email: ronan.sauleau@univ-rennes1.fr).

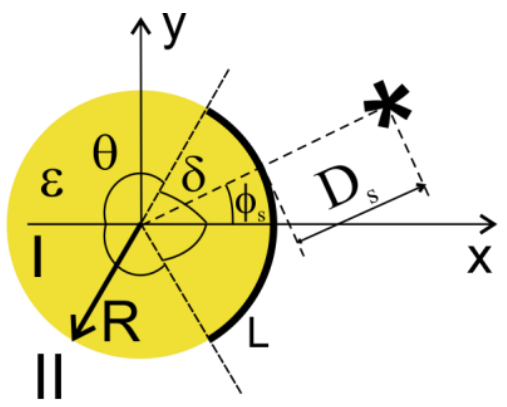

Fig. 1. Cross-section of a dielectric rod with a conformal graphene strip illuminated by a magnetic line current

becomes more intensive and concentrated. This is, in fact, a phenomenon of imperfect near-field focusing, which can be explained, in essence, using the geometrical optics (GO). Hence, it is broadband and, in the 2-D case, takes place both in the E-polarization case (electric field is parallel to the rod) and $\mathrm{H}$-polarization one (magnetic field is parallel to the rod). Note that the focusing can be enhanced greatly by making the sphere of rod discrete, i.e. made of concentric layers, with their dielectric constants mimicking the Luneburg lens $[3,4]$.

According to the reciprocity theorem of classical electromagnetics, if a point source is placed into EMJ area then, in the far zone, a sharp lobe of the angular radiation pattern appears. This far-field collimation can be also called "inverse EMJ" effect; in 2-D, it was studied in [5].

Besides of the GO effects, any finite-size dielectric object is an open resonator, which possesses a discrete spectrum of natural modes, The Pointing theorem tells that such mode frequencies can be only complex valued, i.e. the associated Qfactors are always finite, because of the radiation losses. Out of all dielectric objects, those, which are circular at least in some cross-section, stand out, supporting the whisperinggallery (WG) modes. In ideal sphere and circular rod, WG modes have Q-factors, exponentially growing up with radius, or mode index, that kills performance of rough commercial codes [6-8]. Note that both EMJ and inverse-EMJ effects on finite-radius rod are spoiled by the "ignition" of a WG mode because its near field starts dominating over the GO field [5].

Today, nanotechnologies open new opportunities in the design of many optical and photonics devices, and frequently this is connected to the use of graphene. Besides of the DC 
tunability of its electron conductivity defined by the chemical potential, frequency, temperature, and relaxation time, a major feature of micro and nanosize graphene samples is their ability to support plasmon modes in the $\mathrm{THz}$ to infrared ranges $[9,10]$. Both flat and curved graphene strips attract attention today as easily fabricated tunable components of plasmonic antennas, waveguides, and sensors [11-15]. Recently, in [16] it was shown that a resonance graphene strip placed into the EMJ area of a circular dielectric rod enhances greatly the near field without increasing the radius or refractive index.

Guided by the reciprocity principle, in this work we consider the radiation a $\mathrm{THz}$ range line magnetic current in the presence of a circular dielectric rod, decorated with a conformal strip of graphene. Our aim is to study the inverseEMJ effect, i.e. the far-field directivity enhancement and how can it be controlled with the aid of graphene strip. In our analysis, we combine the Maxwell boundary-value problem with the Kubo conductivity of graphene, considered as a zerothickness layer with complex surface impedance [9].

As it was pointed out to earlier [17,18], such a study is equivalent to accurate quantification of the Purcell effect, i.e. the modification of the power, radiated by a point source (in 2D) in the presence of composite open cavity: dielectric rod with graphene strip. Note that description of this effect with the aid of a simplified quantity called "Purcell factor" [18] is not accurate and should be replaced with numerical analysis.

As circular rod is a simple shape, the center of gravity of a trusted numerical technique is associated with consideration of the graphene strip. Commercial code treatments of zerothickness strip meet certain difficulties (see Introduction in [19]), however, several convergent methods, developed earlier to study the scattering from perfectly electrically conducting (PEC) strips are available. Among them, we have two analytical regularization techniques: regularizing method-ofmoments and Riemann-Hilbert Problem (RHP) method, and a Nystrom-type discretization with Chebyshev quadratures [1921]. The use of these mathematically justified methods allows solving the considered problems quickly and with controlled accuracy even in the sharp WG-mode resonances.

Important examples of such analyses cover flat graphene strip scattering in $[12,14]$. Plasmon-mode resonances in the scattering and absorption by the gratings of coplanar graphene strips were analyzed in [19-21]. The accurately studied curved graphene configurations are restricted to fully covered circular dielectric rod [22-24] and parabolic reflector in air [25].

In our analysis, we use the technique of [16], extensively validated there and adapted here to the line current excitation.

\section{MATHEMATICAL Model}

Consider a cylindrical time-harmonic $\left(e^{-i \omega t}\right)$ wave emitted by a magnetic-line current located near to a circular dielectric rod, the outer boundary of which is partially covered with conformal strip of graphene. The cross section of such antenna configuration by the coordinate plane $z=0$ is presented in Fig. 1. Here, $R$ is the radius of the rod, $2 \delta$ is the angular width of the graphene strip, $2 \theta=2 \pi-2 \delta$ is the angular width of the slot, and $D_{s}$ is the distance from the source to the rod. The electromagnetic field is $\mathrm{H}$-polarized.

Considering the case of $H_{z}$ field not depending on $z$, we obtain the following 2-D problem. Find the function $H_{z}(r, \phi)$, which satisfies (i) the Helmholtz equation with the wavenumber $k^{I \text {, }}$ or $k^{I I}$ for all $r \neq R$, (ii) the dual boundary condition at $r=R$ : on the strip arc, $L:\left\{r=R,\left|\phi-\phi_{0}\right| \leq \delta\right\}$,

$$
E_{\phi}^{I}+E_{\phi}^{I I}=2 Z Z_{0}\left(H_{z}^{I}-H_{z}^{I I}\right), E_{\phi}^{I}={ }_{\phi}^{I I},
$$

and on the slot arc, $S:\left\{r=R,\left|\phi-\phi_{0}\right| \leq \theta\right\}$,

$$
H_{z}^{I}=H_{z}^{I I}, \quad E_{\phi}^{I}={ }_{\phi}^{I I}
$$

(iii) the Sommerfeld radiation condition at infinity, and (iv) the local power finiteness condition (a.k.a. edge condition).

Here, $Z Z_{0}=1 / \sigma$ is the graphene surface impedance, $\sigma$ is the surface conductivity, $Z_{0}$ is the impedance of the free space, index $I(I I)$ is assigned to the inside (outside) domain filled with nonmagnetic material having permittivity $\varepsilon_{I}\left(\varepsilon_{I I}\right)$, and $k_{I, I I}=(\omega /) \sqrt{\varepsilon_{I I I}}$, where $c$ is the speed of light.

The incident cylindrical wave, $H_{z}^{i n c}=H_{0}^{(1)}\left(k^{I}\left|\vec{r}-\vec{r}_{s}\right|\right)$, is emitted by the magnetic line current, placed at the point $\vec{r}_{s}$.

$$
H_{0}\left(k\left|\vec{r} \quad \vec{r}_{s}\right|\right)=\left\{\begin{array}{l}
\sum_{n=-\infty}^{+\infty} J_{n}(k r) H_{n}\left(k r_{s}\right) e^{i n\left(\phi-\phi_{s}\right)}, R<r<r_{s}, \\
\sum_{n=-\infty}^{+\infty} J_{n}\left(k r_{s}\right) H_{n}(k r) e^{i n\left(\phi-\phi_{s}\right.}, r>r_{s},
\end{array}\right.
$$

The total field can be presented as the Fourier series

$$
H_{z}^{(I, I I)}=\left\{\begin{array}{l}
\sum_{n=-\infty}^{+\infty} C_{1, n} J_{n}\left(k^{I} r\right) e^{i n \varphi} / d_{n}, r<R, \\
H_{z}^{i n c}+\sum_{n=-\infty}^{+\infty} C_{2, n} H_{n}\left(k^{I I} r\right) e^{i n \varphi} / h_{n}, r \quad R,
\end{array}\right.
$$

Here, $d_{n}=J_{n}^{\prime}\left(k^{I} R\right), h_{n}=H_{n}^{\prime}\left(k^{I I} R\right), \quad J_{n}(\cdot)$ and $H_{n}(\cdot)$ are the Bessel and Hankel first kind functions, the prime means differentiation in argument, and $C_{(1,2), n}$ are unknown coefficients, which should be found. Such a field satisfies the Helmholtz equation and the radiation condition.

Following [16], we derive a dual series equation for the unknown coefficients $x_{q}$, linearly related to $C_{(1,2), q}$. Using the parametric representations [26] of the integral operators with hyper-singular and logarithmic kernels, we reduce it to a boundary hyper-singular integral equation. Applying a Nystrom-type method with the Chebyshev quadratures, we discretize that equation as follows:

$$
x_{q}+\sum_{p=1}^{N} A_{q p} x_{p}=b_{q}, \quad q=1, \ldots, N,
$$

where $b_{q}=-f\left(t_{0 q}\right) / Z_{q}$ are known, $Z=i \delta Z\left(1-t_{0 q}^{2}\right)$, and the elements of the matrix take the form,

$$
\begin{aligned}
& A_{q q}=Z_{q}^{-1}\left\{-\frac{1}{2} B_{1}(N+1)^{2}-B_{2} \delta^{2}\left[1-\left(t_{0 q}\right)^{2}\right] \times\right. \\
& {\left[\ln 2+2 \sum_{s=1}^{N} T_{s}\left(t_{0 q}\right) T_{s}\left(t_{0 q}\right) / s+(-1)^{2 q} /(2 N+2)\right]-} \\
& \left.2 \delta\left(1-t_{0 q}{ }^{2}\right) K\left(t_{0 q}, t_{0 q}\right)\right\} /\left(\begin{array}{ll}
N & 1
\end{array}\right),
\end{aligned}
$$




$$
\begin{aligned}
& A_{q p}=Z_{q}^{-1}\left\{B_{1}\left[1-\left(t_{0 p}\right)^{2}\right] \frac{1-(-1)^{q+p}}{\left(t_{0 q}-t_{0 p}\right)^{2}}-B_{2} \delta^{2}\left[1-\left(t_{0 p}\right)^{2}\right] \times\right. \\
& {\left[\ln 2+2 \sum_{s=1}^{N} T_{s}\left(t_{0 p}\right) T_{s}\left(t_{0 q}\right) / s+(-1)^{p+q} /(2 N+2)\right]-} \\
& 2 \delta\left[\begin{array}{lll}
1 & \left(t_{0 p}\right)^{2} & \left.K\left(t_{0 q}, t_{0 p}\right)\right\} /(N+1),
\end{array}\right.
\end{aligned}
$$

Using (3), the right part can be written as follows:

$$
\begin{gathered}
f(\phi)=\sum_{n=-\infty}^{\infty} J_{n}^{\prime} H_{n}\left(k r_{s}\right) H_{n}\left(H_{n}^{\prime}\right)^{-1} W_{n} e^{-i n \phi_{s}} e^{i n \phi}- \\
\sum_{n=-\infty}^{\infty} J_{n} H_{n}\left(k r_{s}\right) W_{n} e^{-i n \phi_{s}} e^{i n \phi}
\end{gathered}
$$

where the omitted arguments of Bessel and Hankel functions are, respectively, $k^{I} R$ and $k^{I I} R$, and other quantities are

$$
\begin{gathered}
K\left(t, t_{0}\right)=\sum_{n=1}^{\infty}\left(W_{n}-B_{1}|n|-B_{2}|n|^{-1}\right) \cos n(\theta-\pi)\left(t-t_{0}\right), \\
W_{n}=\left\lfloor\sqrt{I} J_{n}\left(J_{n}\right)^{1}-\sqrt{\varepsilon_{I I}} H_{n}\left(H_{n}^{\prime}\right)^{-}\right\rceil^{-1}, \\
B_{1}=1 / k R\left(\varepsilon_{I}+\varepsilon_{I I}\right), \quad B_{2}=-\frac{1}{2} k R\left(\varepsilon_{I}+\varepsilon_{I I}^{2}\right)\left(\varepsilon_{I}+\varepsilon_{I I}\right)^{-2}
\end{gathered}
$$

Besides, $T_{s}\left(t_{0 q}\right.$ is Chebyshev polynomial of the first kind, and $t_{0 q}$ is zero of Chebyshev polynomial of the second kind.

The matrix equation (5) has strict diagonal predominance. The convergence of solution of (5) with $N \rightarrow \infty$ is guaranteed by the theorems of approximation of hyper-singular operators with the aid of quadratures [27,28].

Further we compute and discuss several characteristics, which depend on the frequency and size of the graphene strip. In the far zone, the total field is a cylindrical wave

$$
H_{z}(\vec{r})=\Phi(\phi)(1 / i \pi k r)^{1 / 2} e^{i k r}
$$

with the following parts of the angular radiation pattern,

$$
\begin{gathered}
\Phi(\phi)=\Phi_{i n}(\phi)+\Phi_{s c}(\phi), \quad \Phi_{i n}(\phi)=e^{i k r \cos \left(\phi-\phi_{0}\right)}, \\
\Phi_{s c}(\phi)=\sum_{n=-\infty}^{+\infty}(-i)^{n} h_{n}^{-1} C_{2, n} e^{i n \phi}
\end{gathered}
$$

The total radiation power is, by definition,

$$
P_{\text {rad }}=\left(k Z_{0}\right)^{-1} \int_{0}^{2 \pi}|\Phi(\phi)|^{2} d \phi
$$

and then the substitution of (13) and (14) leads to

$$
\left.P_{\text {rad }} / P_{0}=1+\sum_{n=-\infty}^{+\infty}\left|y_{n}\right|^{2}+2 \operatorname{Re}\left(y_{n}{ }^{*} J_{n}\left(k r_{s}\right) e^{-i n \phi_{s}}\right)\right],
$$

where $y_{n}=\left(-i^{n} h_{n}^{-1} C_{2, n}\right.$ and the normalization constant is the radiation power of the line in free space, $P_{0}=2\left(k Z_{0}\right)^{-1}$.

The absorption power consists of two parts, corresponding to the power, absorbed in the lossy graphene strip,

$$
P_{a b s}^{g r p h}=\operatorname{Re} Z R \int_{-\delta}^{+\delta}|v(\phi)|^{2} d \phi
$$

and the power absorbed in the lossy dielectric rod,

$$
\left.P_{a b s}^{\text {diel }}=2 \pi R\left|\varepsilon_{I}\right|^{-1} \sum_{m=-\infty}^{\infty}\left|C_{1, m}\right|^{2} \operatorname{Im}_{\lfloor} \sqrt{\varepsilon_{I}} J_{m}\left(k^{I} R\right) J_{m}^{\prime}\left(k^{I^{*}} R\right)\right\rfloor
$$

Thus, the radiation efficiency (the quantum yield efficiency in the language of the Purcell effect) is the ratio,

$$
\eta=P_{r a d} /\left(P_{r a d}+P_{a b s}^{g p h}+P_{a b s}^{\text {diel }}\right)
$$

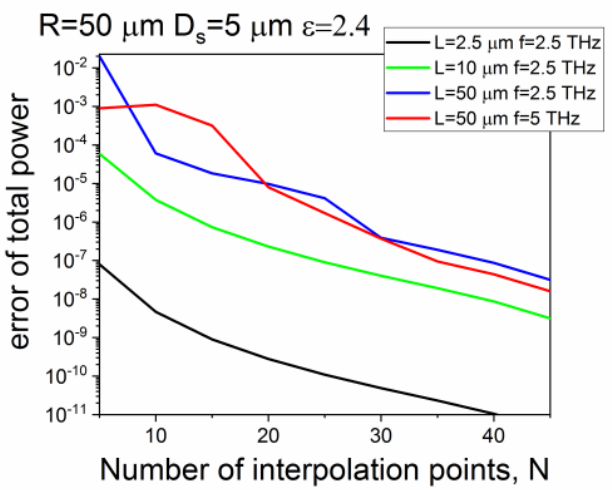

Fig. 2. The far-field computation error as a function of the order of interpolation in the Nystrom type discretization.

The far-field directivity is the ratio of the power, radiated in the main-lobe direction, $\phi_{\max }$, to the total radiated power,

$$
D=2\left(k Z_{0} P_{\text {rad }}\right)^{-1}\left|\left(\phi_{\max }\right)\right|^{2},
$$

Note that the line-current directivity in the free space is 1.

Important part of the modeling is the description of the surface impedance of graphene. We use the Kubo formalism [9], according to which the graphene surface impedance, up to the visible-light frequencies, is given by

$$
Z(\omega) \approx\left(Z_{0} \sigma_{\text {intra }}\right)^{-1}=(1 / \tau-i \omega) /\left(Z_{0} c_{1}\right),
$$

where

$$
c_{1}=q_{e}^{2} k_{B} T\left(\pi \hbar^{2}\right)^{-1}\left\{\mu_{c}\left(k_{B} T\right)^{-1}+2 \ln \left(1+\exp \left(-\mu_{c}\left(k_{B} T\right)^{-1}\right)\right)\right\},
$$

$q_{e}$ is the electron charge, $k_{B}$ is the Boltzman constant, $T$ is the temperature, $\hbar$ is the reduced Planck constant, $\tau$ is the electron relaxation time and $\mu_{\mathrm{c}}$ is the chemical potential.

As known, a strip of graphene can be considered as a plasmon surface wave Fabry-Perot resonator [12,16,21]. If $|Z| \gg 1$, and neglecting the strip curvature, the approximate empiric equation for the plasmon modes is obtained as

$$
\begin{gathered}
\sin \left(\operatorname{Re} \gamma_{\text {plas }} 2 \delta R+\psi\right) \approx 0, \\
\left.\gamma_{\text {plas }} \approx k\left[\left(\varepsilon_{I}+\varepsilon_{I I}\right) / 2\right]^{1 / 2} 1-Z\left(\varepsilon_{I}+\varepsilon_{I I}\right)+O\left(Z^{-2}\right)\right]
\end{gathered}
$$

Here, $\psi$ is the phase of the coefficient of reflection of the plasmon wave from the strip edge; the best fit is $\psi=\pi / 4$.

The roots of (22) determine the natural frequencies of the plasmon modes $P_{m}$, whose fields are symmetric $(m=1,3, \ldots)$ and anti-symmetric $(m=2,4, \ldots)$ w.r.t. the strip middle point,

$$
\omega_{m} \approx \frac{1}{2}(\pi m-\psi) Z_{0} c c_{1}\left[\left(\varepsilon_{I}+\varepsilon_{I I}\right) \delta R\right]^{-1}+O\left(Z^{-2}\right) .
$$

\section{NUMERICAL RESULTS}

First of all, to check the convergence we compute the error,

$$
e_{P}(N)=\left|P_{r a d}(N) \quad P_{r a d}\left(N_{\max }\right)\right| / P_{r a d}\left(N_{\max }\right)
$$

in the far-field power, as a function of the discretization order, with respect to the power computed at $N_{\max }=50$. These plots are presented in Fig. 2 and serve as the code validation. The antenna parameters are explained in the inset. 


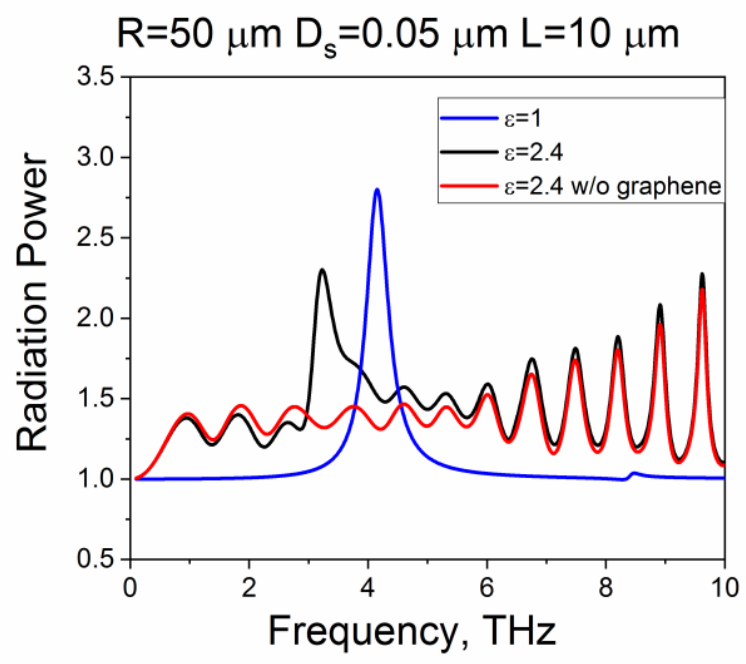

(a)

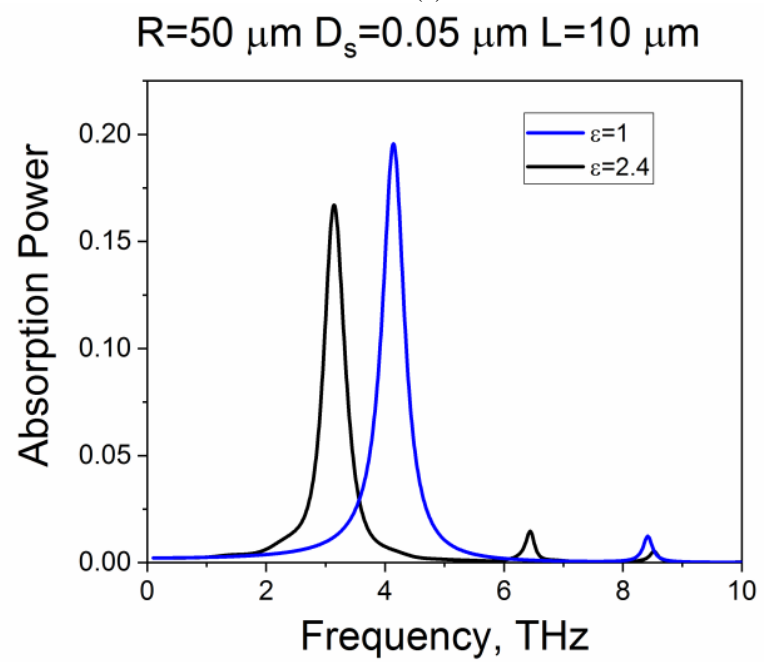

(b)

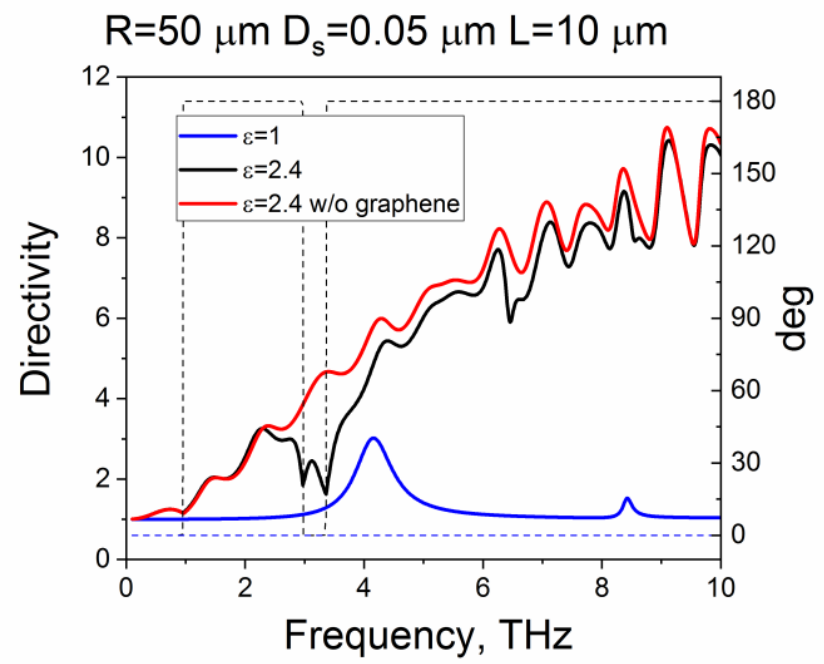

(c)

Fig. 3. Spectra of normalized radiation (a) and absorption power (b) and directivity (c) as a function of the frequency for the dielectric rod without grapheme strip (red), with grapheme strip (black), and for the grapheme strip in the free space (blue). The dotted line on panel (c) depicts the main lobe radiation angle. Dielectric rod has radius of $R=50 \mu \mathrm{m}$ and permittivity $\varepsilon=2.4$. Graphene width is $L=10 \mu \mathrm{m}$. The source is on the $x$-axis at the distance $D_{s}=50 \mathrm{~nm}$ from the rod.

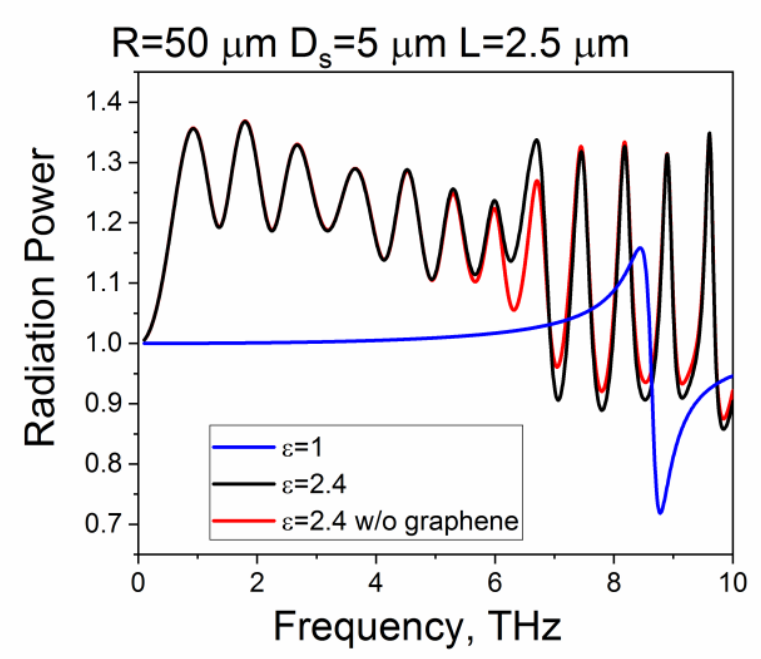

(a)

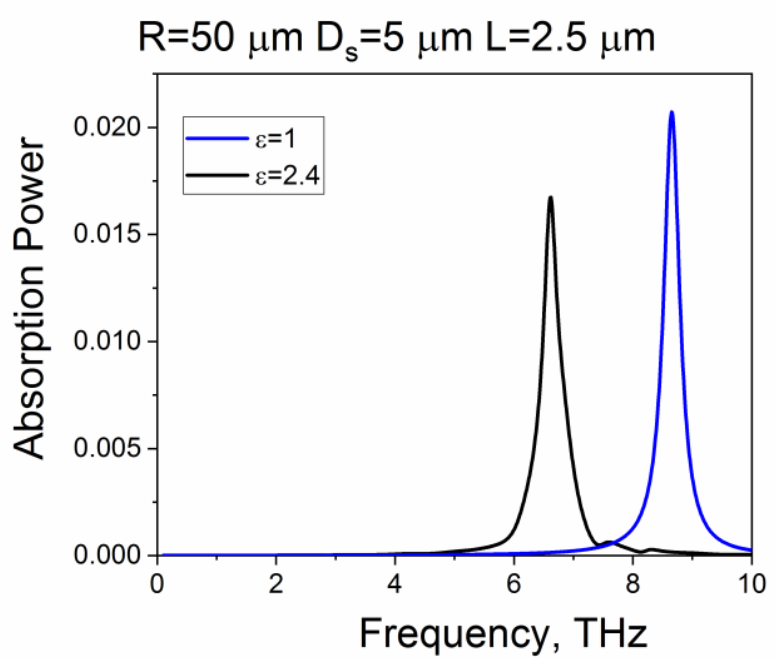

(b)

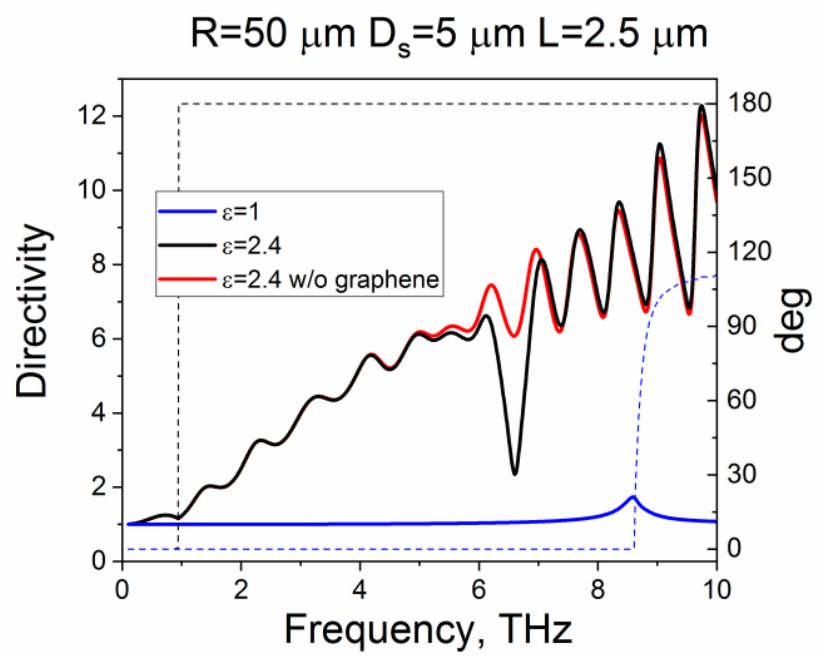

(c)

Fig.4. The same in Fig. 3, however for $\quad s \quad 5 \mu \mathrm{m}, L=2.5 \mu \mathrm{m}$

At first, we assume that the rod is lossless and $P_{a b s}^{\text {diel }}=0$. In Figs. 3 to 5 , we present the spectral dependences, up to 10 $\mathrm{THz}$, of (a) radiation power, (b) absorption power, and (c) directivity and the angle of the main lobe, $\phi_{\max }$ (dotted lines). 


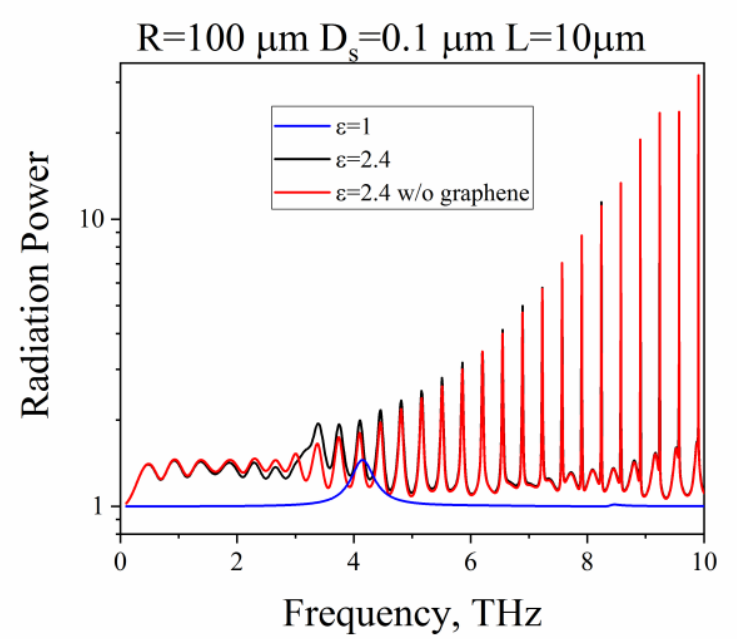

(a)

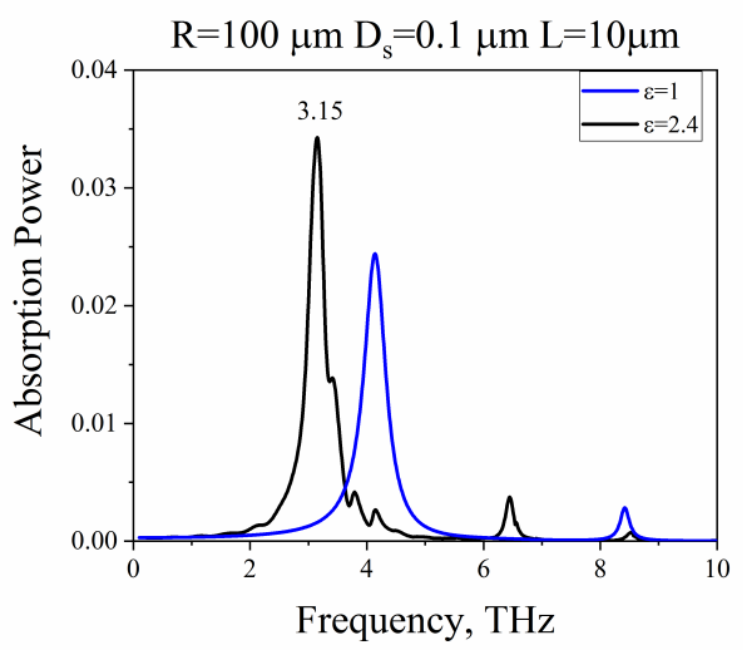

(b)

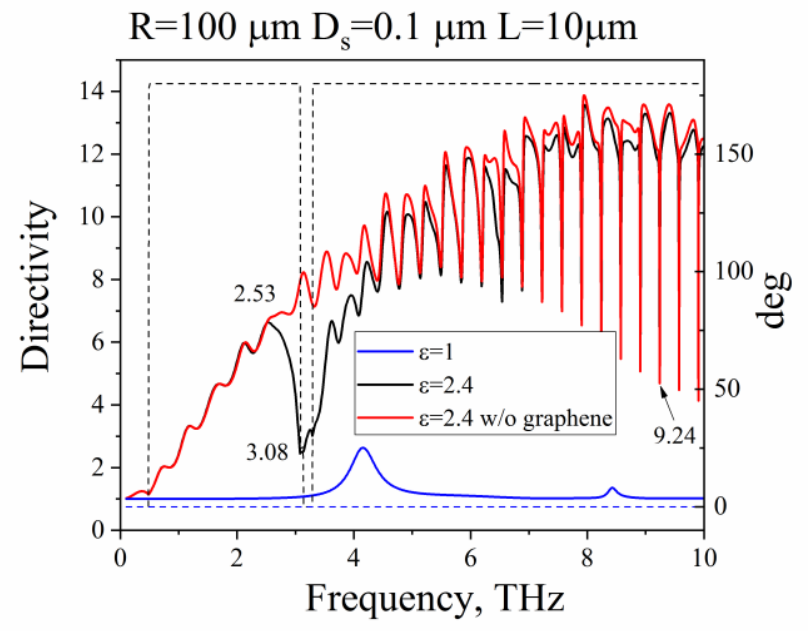

(c)

Fig. 5. The same in Fig. 3, however for $R=100 \mu \mathrm{m}, D_{s}=0.1 \mu \mathrm{m}$.

The parameters of graphene and dielectric are the same for all figures: $\tau=1 \mathrm{ps}, T=300 \mathrm{~K}, \mu_{c}=0.5 \mathrm{eV}$, and $\varepsilon=2.4$.

The source coordinates are taken on the $x$-axis all times.

In Figs. 3 and 4, the rod radius is $R=50 \mu \mathrm{m}$ and the strip width, $L=\delta R$, is different, $10 \mathrm{~mm}$ and $2.5 \mathrm{~mm}$, respectively.

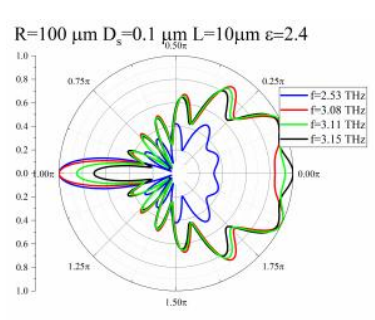

(a)

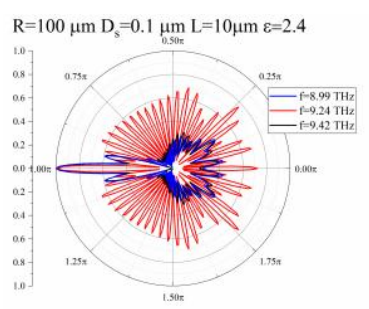

(c)

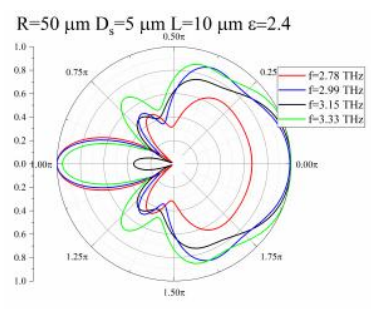

(e)

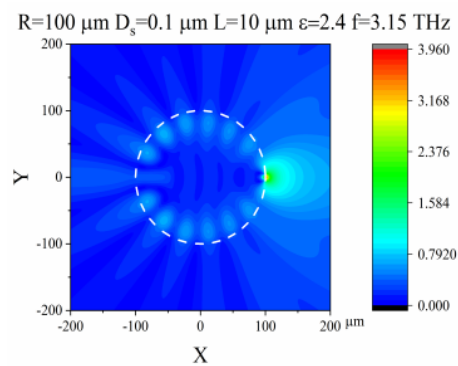

(b)

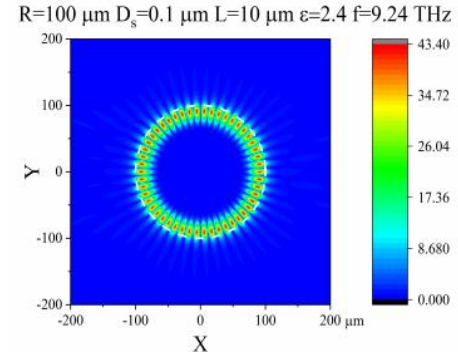

(d)

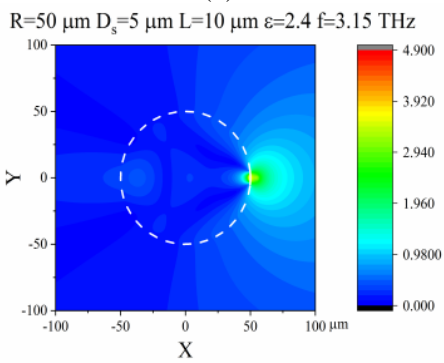

(f)
Fig. 6. Normalized far-field angular radiation patterns in and near to some of the peaks of absorption power in Figs. 3-5, and near field portraits in the peaks.

As the plasmon frequency scales as inverse square-root of strip width (see (24)), in the latter case the plasmon resonance $P_{1}$ shifts to twice higher frequency than in the former case. It can be seen that the excitation of the plasmon mode adds to the radiation power (or Purcell factor) of antenna, however, at the expense of the spike in the absorption power. In the upper$\mathrm{THz}$ range, the resonances on the WG modes appear, which are absent on the absorption plots because the rod is assumed lossless. A shift of the source from the rod plays little role.

The plots of the directivity of emission on panels (c) reveal very interesting interplay of three effects. One of them has GO nature - this is inverse EMJ effect, which is displayed as a remarkable steady growth of $D$ with frequency from 1 in static limit to some 10 at $10 \mathrm{THz}$. The main lobe looks in the direction of $\phi=180^{\circ}$. However, this growth is spoiled by the plasmon resonance $P_{1}$, accompanied with a deep drop in the directivity. This is because the natural field of the first-order plasmon mode has the "dipole" pattern, with strong backward radiation (i.e. in the direction of $\phi=0$ ). Note that, in the case of wider strip (Fig. 3 (c)), this happens to be enough to "overkeel" the main lobe direction. If the strip is narrower (Fig. 4 (c)), the resonance $P_{1}$ appears at higher frequencies, where the inverse-EMJ effect is stronger, and then no resonance "overkeel" happens. 


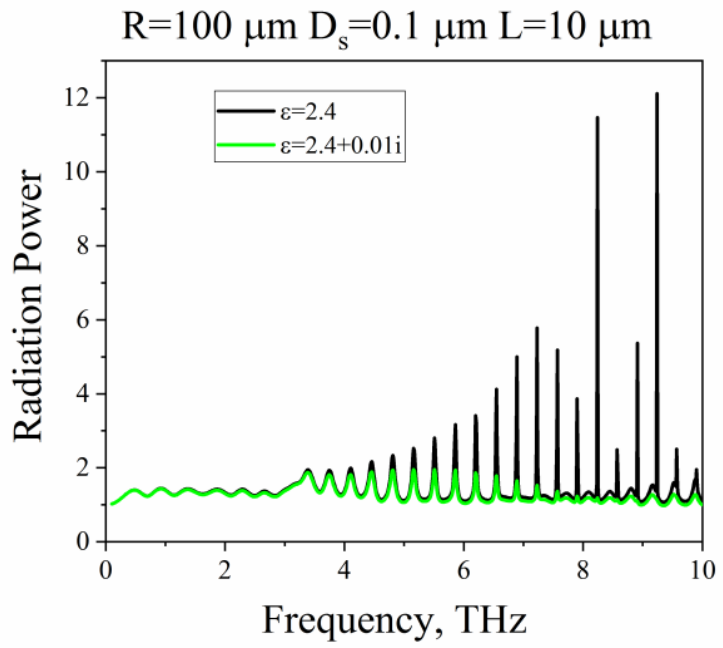

(a)

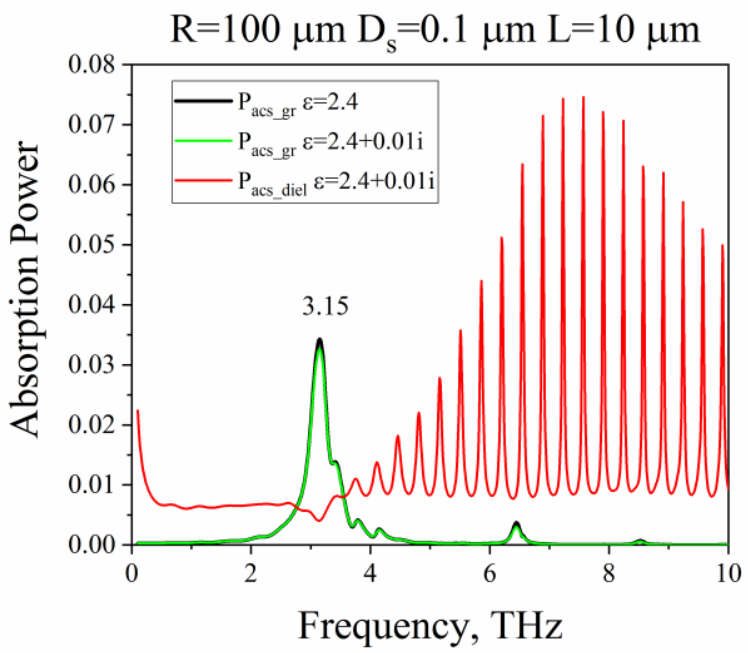

(b)

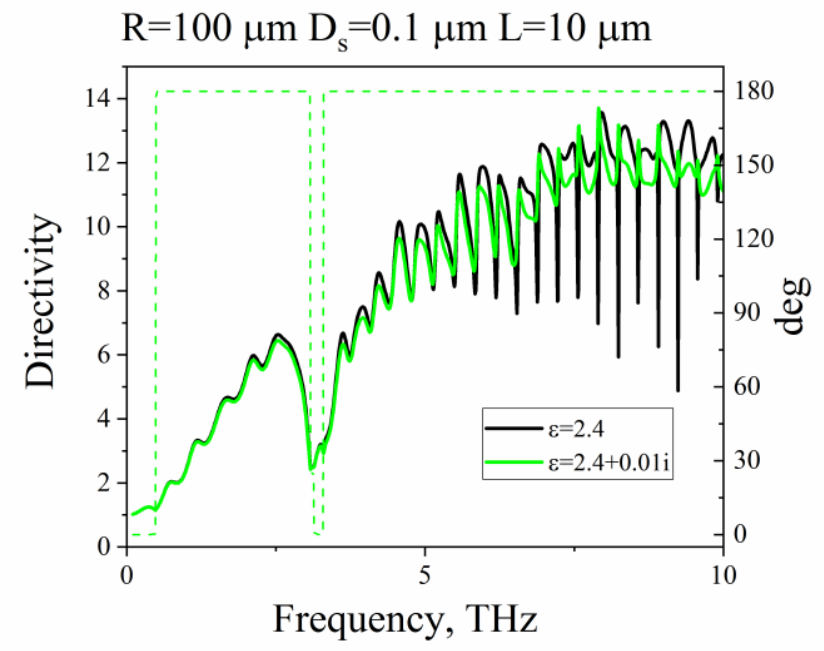

(c)

Fig. 7. The same characteristics as in Fig. 5, however for the lossy dielectric rod, $\varepsilon=2.4+i 0.01$. For comparison, the lossless rod data are also shown.

The plots in Fig. 5 show similar results, however for twice wider rod decorated with wide strip of graphene. In this case, the radiation power (i.e. the full-wave Purcell factor) display a

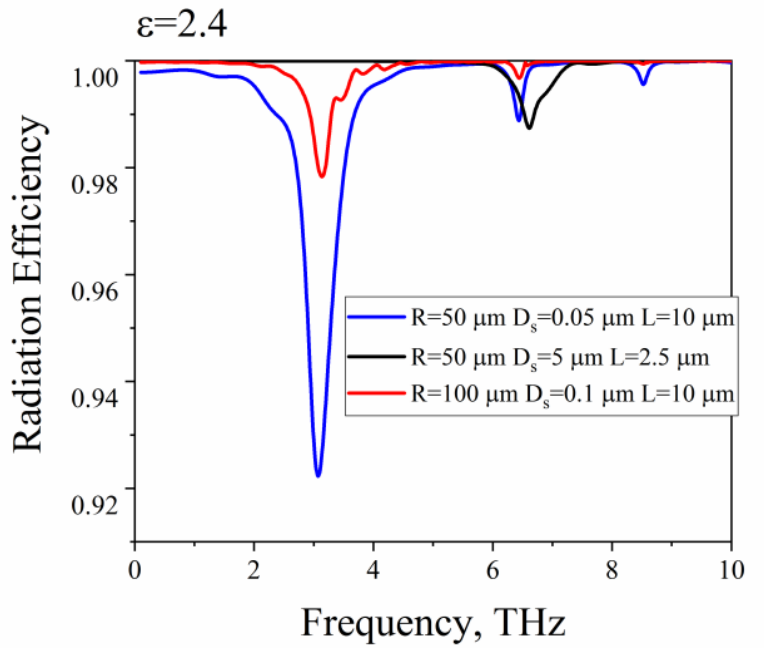

Fig. 8. Radiation efficiency as a function of frequency in the case of lossless dielectric rod. Antenna parameters are explained in the inset.

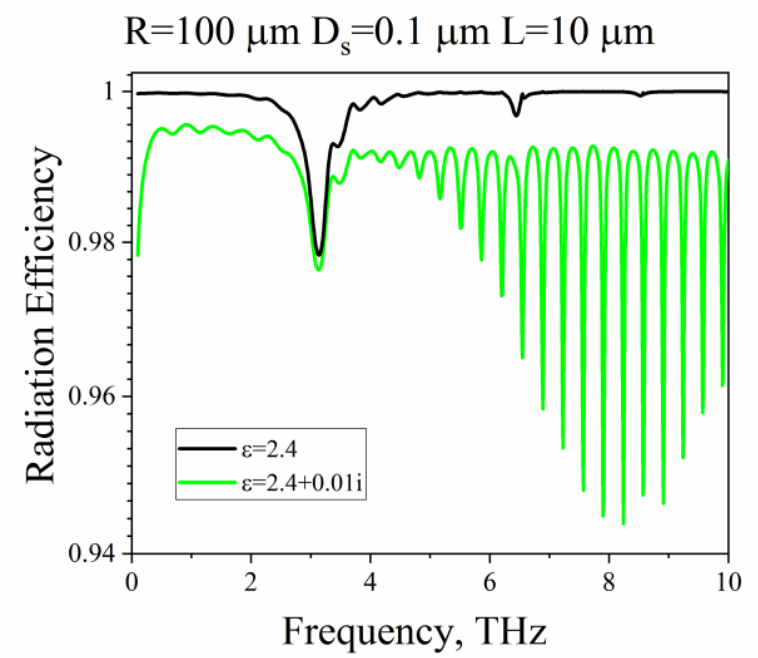

Fig. 9. Comparison of radiation efficiencies versus the frequency in the case of lossless (black) and lossy (green) dielectric rod.

periodic sequence of sharper and sharper spikes at higher frequencies. These are the resonances on the WG modes of the circular rod, $H_{1 m}(m=1,2, \ldots)$, that have Q-factors, exponentially growing with azimuth index $m$. They are absent on the plots of the absorption power as the rod is assumed lossless. They spoil the directivity (i.e. the inverse-EMJ effect) in the same manner as the plasmon-mode resonance, although, unlike the latter, they cannot "over-keel" the main lobe.

To support our understanding of the behavior of the antenna directivity as a function of the frequency, we visualize, in Fig. 6 , three sets of the normalized far-field radiation patterns and the near-field portraits. The former are plotted both in and off the resonances, and the latter are shown only in the resonances. In the case of plasmon resonance, they demonstrate enhanced backward radiation (a), (e) and near field, which sticks to the graphene strip (b), (f). The main lobe, in this resonance, looks backward direction. 


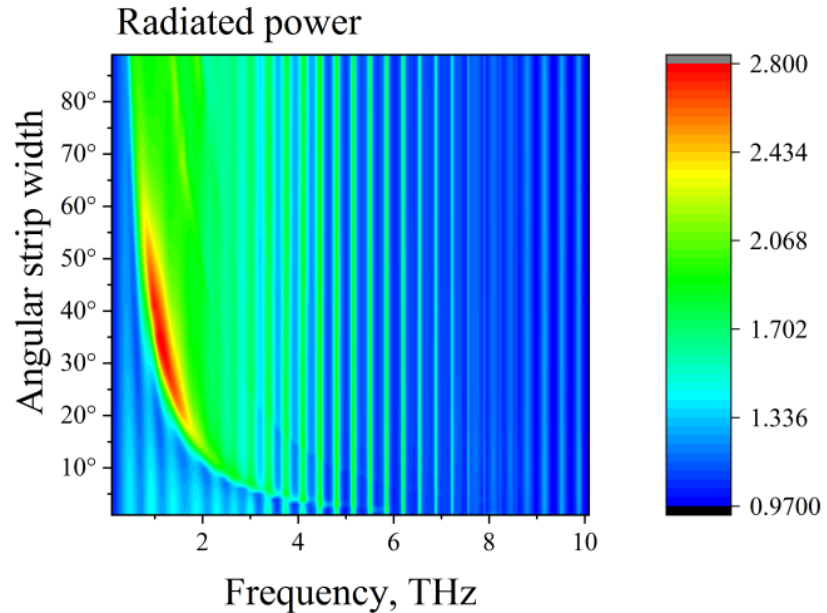

(a)

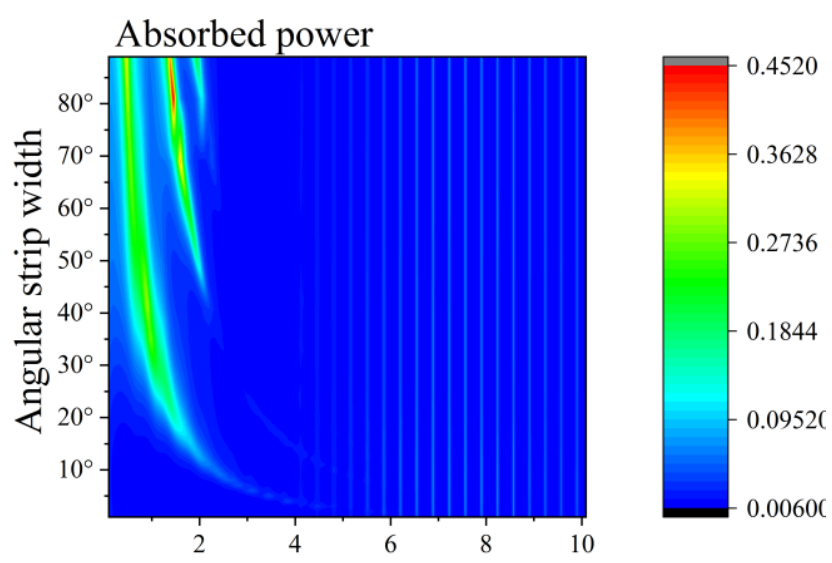

Frequency, THz

(b)

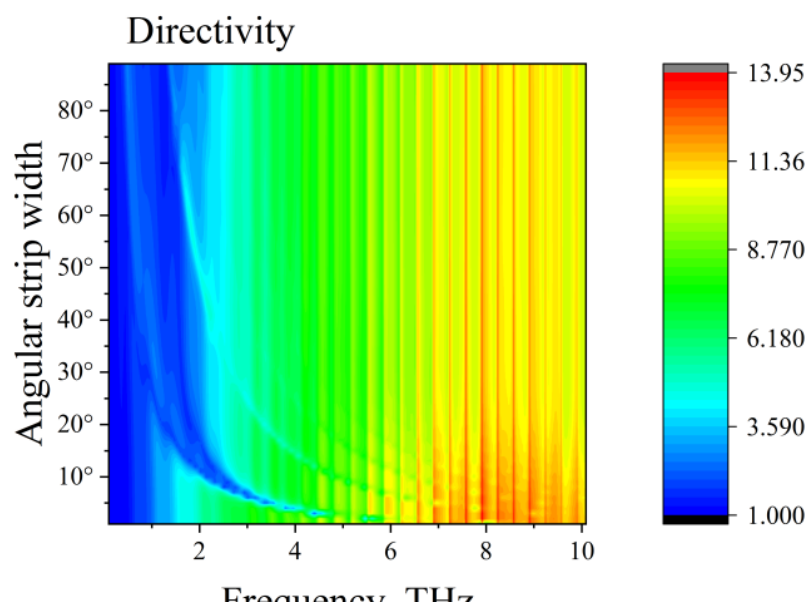

Frequency, $\mathrm{THz}$

(c)

Fig. 10. Color maps of the normalized radiated (a) and absorbed (b) powers and the directivity (c) of emission versus the frequency and the strip angular width, for antenna with the other parameters being the same as in Fig. 9.

In the case of the resonance on the WG-mode $H_{1,25}$, see panels (c) and (d), there are as many as 50 comparable lobes in the far-field pattern and 50 bright spots in the near field, inside the dielectric rod. Still, the main lobe of radiation looks in the forward direction even in the resonance.
Keeping in mind that even the best of available dielectric materials are lossy, we have also checked how these losses affect the above discussed antenna characteristics. In the language of the Purcell effect, this is equivalent to account of non-radiation decay rate due to both dielectric and graphene losses. The corresponding results are presented in Fig. 7 for the case of $\varepsilon=2.4+i 0.01$. They show that the plasmon-mode resonance and associated drop in the directivity is almost intact. However, now the WG-modes appear as small but sharp spikes in the normalized absorption power. The plots of the radiation power and the directivity become smoother because the Q-factors of WG modes are now spoiled.

Finally, we discuss the radiation efficiency of the studied photonic antenna. This is the same as quantum yield efficiency in terms of the Purcell effect. The frequency dependences of this quantity are presented in Figs. 8 and 9 for the lossless rod and lossy rod, respectively. As could be expected, the radiation efficiency drops in the plasmon resonances, however, this drop is only a few per cent as it has the order of $O(\operatorname{Re} Z)$. In similar manner, if the rod is lossy then the efficiency drops in the WG-mode resonances; here the drops have the order of $O(\operatorname{Im} \varepsilon)$.

More complete understanding of the variation of the above discussed characteristics with frequency and strip size, for the other parameters taken as in Fig. 9, is provided by the color maps in Fig. 10. Here, one can easily discriminate between the WG-mode resonances, which have fixed frequencies, and plasmon-mode resonances, which follow equation (24).

\section{CONCLUSIONS}

We have presented the results of the accurate study of the modification of the H-polarized radiation emitted by magnetic line current, due to isotropic circular dielectric rod decorated with graphene strip. Our results, obtained by a mathematically grounded in-house algorithm based on judicious solution of the hypersingular integral equation, have shown that such antenna is able to produce well-collimated radiation in the far zone, due to the inverse EMJ effect. However, this broadband collimation is spoiled if the frequency hits one of the natural frequencies, which are of two kinds: of the medium-Q stripplasmon modes and of the extremely high-Q rod WG modes.

What appears to be important for applications, only the plasmon-mode resonances are tunable with the aid of DC bias, which changes the chemical potential of graphene. This makes possible electrically controlled binary operation of antenna at a fixed frequency, via the main lobe blinking in the forward direction. Note that if the strip were a PEC one, then the plasmon modes and the associated resonances would be absent. In this case, the strip mostly screens the rod from the source and the EMJ effect is spoiled.

We would like to emphasize that our code, besides of the controlled accuracy, is very fast: the plots and maps presented above are computed within dozens of minutes on a moderate desktop computer. 


\section{REFERENCES}

[1] B. S. Lukyanchuk, R. Paniagua-Domínguez, I. Minin, O. Minin, and Z. Wang, "Refractive index less than two: photonic nanojets yesterday, today and tomorrow," Opt. Mat. Exp., vol. 7, no 6, pp. 1820-1847, 2017.

[2] A. Heifetz, S.-C. Kong, A. V. Sahakian, et al., "Photonic nanojets," J. Comput. Theor. Nanosci., vol. 6, no. 9, pp. 1979-1992, 2009.

[3] R. K. Luneburg, The Mathematical Theory of Optics. Providence, RI: Brown Univ. Press, 1941.

[4] J. A. Lock, "Scattering of an electromagnetic plane wave by a Luneburg lens. III. Finely stratified sphere model," J. Opt. Soc. Am. A, vol. 25, no 12, pp. 2991-3000, 2008.

[5] A.V. Boriskin and A.I. Nosich, "Whispering-gallery and Luneburg lens effects in a beam-fed circularly-layered dielectric cylinder," IEEE Trans. Antennas Propagat., vol. 50, no 9, pp. 1245-1249, 2002

[6] G. L. Hower, R. G. Olsen, D. J. Earls, and J. B. Schneider, "Inaccuracies in numerical calculation of scattering near natural frequencies of penetrable objects," IEEE Trans. Antennas Propagat., vol. 41, no 7, pp. 982-986, 1993.

[7] A. V. Boriskin, S. V. Boriskina, A. Rolland, R. Sauleau, and A. I. Nosich, "Test of the FDTD accuracy in the analysis of the scattering resonances associated with high- $Q$ whispering-gallery modes of a circular cylinder," J. Opt. Soc. Am. A, vol. 25, no 5, pp. 1169-1173, 2008.

[8] J. Niegemann, W. Pernice, and K. Busch, "Simulation of optical resonators using DGTD and FDTD," J. Opt. A, Pure Appl. Opt., vol. 11, no 11, p. $114015 / 10,2009$.

[9] G. W. Hanson, "Dyadic Green's functions and guided surface waves for a surface conductivity model of graphene," J. Appl. Phys., vol. 103, pp. 064302, 2008

[10] M. Jablan, H. Buljan, and M. Soljacic, "Plasmonics in graphene at infrared frequencies," Phys. Rev. B, vol. 80, p. 245435, 2009.

[11] M. Yasir and P. Savi, "Commercial graphene nanoplatelets-based tunable attenuator," Electron. Lett., vol. 56, no 4, pp. 184-187, 2020.

[12] O. V. Shapoval and A. I. Nosich, "Bulk refractive-index sensitivities of the THz-range plasmon resonances on a micro-size graphene strip," $J$. Phys. D: Appl. Phys., vol. 49, no 5, pp. 055105/8, 2016.

[13]A. P. Anyutin, I. P. Korshunov, and A. D. Shatrov, "Quasi-static plasmon resonances in a graphene ribbon in the infrared range," J. Communic. Technol. Electron., vol. 61, no. 6, pp. 607-613, 2016.

[14]W. Fuscaldo, P. Burghignoli, P. Baccarelli, and A. Galli, "Efficient 2-D leaky-wave antenna configurations based on graphene metasurfaces," Int. J. Microw. Wireless Techn., vol. 9, no 6, pp. 1293-1303, 2017.

[15]O. V. Shapoval, et al., "Integral equation analysis of plane wave scattering by coplanar graphene-strip gratings in the THz range," IEEE Trans. Terahertz Science Technol., vol. 3, no 5, pp. 666-673, 2013.

[16]S. V. Dukhopelnykov, R. Sauleau, M. Garcia-Vigueras, and A. I. Nosich, "Combined plasmon-resonance and photonic-jet effect in the $\mathrm{THz}$ wave scattering by dielectric rod decorated with graphene strip," J. Appl. Phys., vol. 126, no 2, art. no 023104, 2019.

[17]M. V. Balaban, R. Sauleau, T. M. Benson, and A. I. Nosich, "Accurate quantification of the Purcell effect in the presence of a microdisk of nanoscale thickness," Micro Nano Lett., vol. 6, no 6, pp. 393-396, 2011.

[18]A. E. Krasnok, et al., "An antenna model for the Purcell effect," Sci. Rep., vol. 5, art no 12956, 2015.

[19]T. L. Zinenko, "Scattering and absorption of terahertz waves by a freestanding infinite grating of graphene strips: analytical regularization analysis," J. Opt., vol. 17, no 5, pp. 055604/8, 2015.

[20]M. E. Kaliberda, L. M. Lytvynenko, S. A. Pogarsky, and M. P. Roiuk, "Diffraction of the H-polarized plane wave by a finite layered graphene strip grating," Int. J. Microwave Wireless Technol., vol. 11, 2019.

[21]T. L. Zinenko, A. Matsushima, and A. I. Nosich, "Surface-plasmon, grating-mode and slab-mode resonances in $\mathrm{THz}$ wave scattering by a graphene strip grating embedded into a dielectric slab," IEEE J. Sel. Top. Quant. Electron., vol. 23, no 4, art. no 4601809, 2017.

[22]M. Riso, M. Cuevas and R. A. Depine, "Tunable plasmonic enhancement of light scattering and absorption in graphene-coated subwavelength wires," J. Opt., vol. 17, pp. 075001/8, 2015.

[23]E. A. Velichko, "Evaluation of a dielectric microtube with a graphene cover as a refractive-index sensor in the THz range," J. Opt., vol. 18, no 3 , pp. $035008 / 11,2016$.

[24]M. Naserpour, C. J. Zapata-Rodríguez, S. M. Vuković, et al., "Tunable invisibility cloaking by using isolated graphene-coated nanowires and dimers," Sci. Rep., vol. 12, pp. 12186/14, 2017.
[25]T. Oguzer, A. Altintas, and A. I. Nosich, "Focusing of THz waves with a microsize cylindrical reflector made of graphene in the free space," $J$. European Opt. Soc., vol. 13, art. no 16, 2017.

[26]Y. V. Gandel, "Parametric representations of integral and pseudodifferential operators in diffraction problems," Proc. Int. Conf. Mathem. Methods Electromag. Theory (MMET-2004), Dnipro, 2004, pp. 57-62.

[27] A. S. Kononenko et al., "Singular and hypersingular integral equations techniques for gyrotron coaxial resonators with a corrugated insert," Int. J. Infrared Millimeter Waves, vol. 28, no 4, pp. 267-274, 2007.

[28]A. V. Kostenko, "Numerical method for the solution of a hypersingular integral equation of second kind," Ukrainian Mathem. J., vol. 65, no. 9, pp. 1373-1383, 2014.

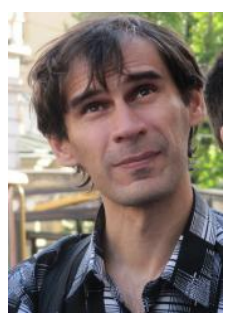

Sergii V. Dukhopelnykov (M'18) was born in Kharkiv, Ukraine in 1982. He received the B.S., M.S. and Ph.D. degrees in mathematical modeling and numerical methods from the Kharkiv National University in 2003, 2004 and 2010, respectively.

From 2007 to 2018, he was a Lecturer, Senior Lecturer and Assistant Professor with the Department of Mathematics, National Technical University "Kharkiv Polytechnic Institute" in Kharkiv, Ukraine. Since 2018, he is Senior Scientist in the Laboratory of Micro and Nano Optics, Institute of Radio-Physics and Electronics NASU, Kharkiv and part-time Assistant Professor at the School of Mathematics of the Kharkiv National University. His research interests are in singular integral equations, Nystrom methods, and patterned graphene scattering. He was recipient of the Ph.D. scholarship award of the N. I. Akhiyezer Foundation, Kharkiv (2010) and the Young Scientist Prize of the International Conference on Mathematical Methods in Electromagnetic Theory (2018).

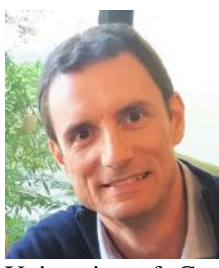

Mario Lucido (M'04-SM'17) was born in Naples, Italy, in 1972. He received the Laurea degree (summa cum laude) in electronic engineering from the University of Napoli "Federico II", Naples, Italy, in 2000 and the Ph.D. degree in electrical and telecommunication engineering from the University of Cassino and Southern Lazio, Cassino, Italy, in 2004.

Since April 2005, he has been a Researcher at the University of Cassino and Southern Lazio, where he has been Adjunct Professor of antennas and microwaves since 2006. Dr. Lucido has authored about 80 scientific journal articles and conference papers. His research interests include semi-analytical techniques for the analysis of scattering problems, waveguide and optical waveguide propagation, microwave circuits and microstrip antennas. Dr. Lucido is currently a Member of the Italian Society of Electromagnetism (SIEM), the National Inter-University Consortium for Telecommunications (CNIT), ELEDIA Research Center, and the National Interuniversity Research Center on Interactions between Electromagnetic Fields and Biosystems (ICEmB). Dr. Lucido received the "Giorgio Barzilai" Prize for the Best Young Scientist Paper at the Italian National Congress on Electromagnetics (RiNEm) in 2006 and the Volodymyr G. Sologub Senior Researcher Award for contribution to the development of analytical regularization methods in computational electromagnetics at the International Conference on Mathematical Methods in Electromagnetic Theory (MMET) in Lviv, 2016.

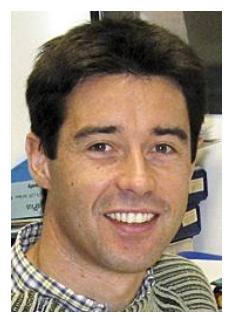

Ronan Sauleau (M'04-SM'06-F'18) was born in Rennes, France in 1972. He received the DEA and Electronic Engineering and Radiocommunications degrees from the Institut National des Sciences Appliquees, Rennes, France, in 1995, the Aggregation degree from Ecole Normale Superieure de Cachan, France, in 1996, and the Doctoral degree in signal processing and telecommunications from the Institut d'Electronique et Telecommunications de Rennes, University of Rennes 1, Rennes, in 1999.

Since 1999, he has been on the staff of IETR, Professor since 2009, and Director since 2017. His current research field is numerical modeling, millimeter-wave printed and reconfigurable antennas, substrate integrated waveguide antennas, lenses, electromagnetic bandgap materials, metamaterials, reflectarrays, and transmitarrays, and biological effects of 
millimeter waves. Prof. Sauleau served as a national delegate for COST VISTA, and he is a member of the board of directors of the European Association on Antennas and Propagation since 2013. He received the 2004 ISAP Conference Young Scientist Travel Grant and the first Young Researcher Prize in Brittany, France, in 2001 for his work on gain-enhanced Fabry-Perot antennas. In 2007, he was elected Junior Member of the Institute Universitaire de France. He was awarded the Bronze medal by CNRS in 2008.

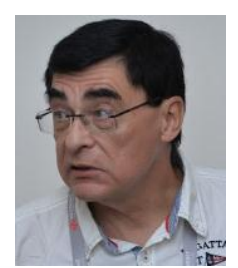

Alexander I. Nosich (M'94-SM'95-F'04) was born in 1953 in Kharkiv, Ukraine. He received the M.S., Ph.D., and D.Sc. degrees in radio physics from the Kharkiv National University, Ukraine, in 1975, 1979, and 1990, respectively.

Since 1979, he has been with the Institute of Radio Physics and Electronics NASU, Kharkiv, where he is currently Professor and Principal Scientist and Head, Laboratory of Micro and Nano Optics. Since 1992, he held many guest fellowships and professorships in the EU, UK, Japan, Singapore, and Turkey. His research interests include the method of analytical regularization, propagation and scattering of waves, open waveguides, antennas and lasers, and the history of microwaves.

Prof. Nosich was initiator and technical committee chairman of the international conference series on Mathematical Methods in Electromagnetic Theory (MMET), held in Ukraine since 1990. In 1995, he organized the IEEE AP-S East Ukraine Chapter, the first one in the former USSR. Currently he represents Ukraine in the European Association on Antennas and Propagation. He was awarded the honorary title of Doctor Honoris Causa of the University of Rennes 1, France (2015) and the Galileo Galilei Medal of the International Commission for Optics (2017), and elected Fellow of the Optical Society (OSA) (2020). He was also co-recipient the 2017 National Prize of Ukraine in Science and Technology for the works entitled, "Photonics of semiconductor and dielectric nanostructures" and the 2018 Solomon I. Pekar Award of NASU in the solid state physics theory. 